\title{
OBSERVATIONAL PROPERTIES OF SYNTHETIC VISUAL BINARY CATALOG
}

\author{
P. Nurmi ${ }^{1}$ \\ RESUMEN
}

Las misiones astrométricas futuras observarán un gran número de nuevas binarias de las cuales muchas serán binarias visuales. Para planear detalladamente los mejores procedimientos para su detección es necesario tener información previa sobre las propiedades observacionales de las binarias visuales esperadas. Por ello, se ha creado y analizado un catálogo de binarias sintéticas. Los resultados nos ayudan a entender los tipos de binarias que se espera encontrar en los catálogos que generarán las misiones astrométricas. Estos resultados representan distribuciones "reales" si todas las binarias fueran observadas. Cualquier proyecto observacional real, al igual que cualquier misión astrométrica, muestrea sólo una pequeña fracción de la población real, dependiendo de la capacidad de óbservación de la misión. En este estudio consideramos solo números relativos con respecto al número total de binarias que se supone que existen en el cielo hasta una magnitud límite, dada por la misión astrométrica.

\section{ABSTRACT}

Forthcoming astrometric missions will observe a huge number of new binaries from which a large fraction will be visual binaries. Detailed planning of optimal detection procedures requires pre-launch information about the observational properties of expected visual binaries. Hence, a synthetic binary catalog is created and analyzed for observational properties of visual binary stars. These results help to understand what kind of binaries we expect to find in the final output catalogs of astrometric missions. These results represent 'true' binary distributions if all of them would be observed. All real observational projects or astrometric satellites sample only small fractions of these populations depending on the observational capabilities of the missions. In this study we consider only relative numbers with respect to the total number of binary stars assumed to exist in the sky down to the magnitude limit depending on the astrometric mission.

\section{Key Words: BINARIES: VISUAL - METHODS: DATA ANALYSIS}

\section{SIMULATION PROCEDURE}

This study is a continuation of an earlier paper (Nurmi \& Boffin 2003), where we used the HST Guide Star Catalogue (GSC) 1.2 together with stellar evolution codes to model the observational properties of visual binary stars down to apparent visual magnitudes $m_{v}<15$. In this paper we do a similar study, but now using GSC 2.2 and hence achieving fainter magnitudes. The details of the simulation method can be obtained from the previous paper (Nurmi \& Boffin 2003).

The main idea is to start the simulation by choosing two masses randomly from the initial mass function (IMF) (Kroupa 2002) and create a binary system by choosing the orbital parameters from the binary distributions. The stars are evolved using stellar evolution algorithms (Hurley, Pols \& Tout 2000) according to their age and metallicity distributions. By varying the distance and stellar properties, we find a match between the trial star and a star at the

\footnotetext{
${ }^{1}$ Royal Observatory of Belgium. email: pasnurmi@oma.be
}

observed $m_{v}$ distribution of GSC 2.2. We repeat the whole procedure for every individual field and then collect the information covering the whole sky. In the simulations $10^{2}$ random fields are chosen from the sky each having a size of $\sim 1$ square degree. It is assumed that every star is a binary and in the final sample we have $\sim 2.5 \times 10^{5}$ binary stars, which is sufficient for our statistical analysis. All stars situated at the galactic plane $|b|<10^{\circ}$ are excluded.

\section{INITIAL BINARY DISTRIBUTIONS}

The orbital parameters of binaries are chosen from the following distributions:

- Inclination $i \propto \sin (i)$

- Eccentricity $e: f(e)=2 e$

- Semimajor-axis $a$ : $\log (a)$ is uniformly distributed between $0.1 \mathrm{AU}$ and $10^{6} \mathrm{AU}$

- Remaining angular elements: $\omega, \Omega$ and $M$ have uniform distribution between 0 and $2 \pi$ 

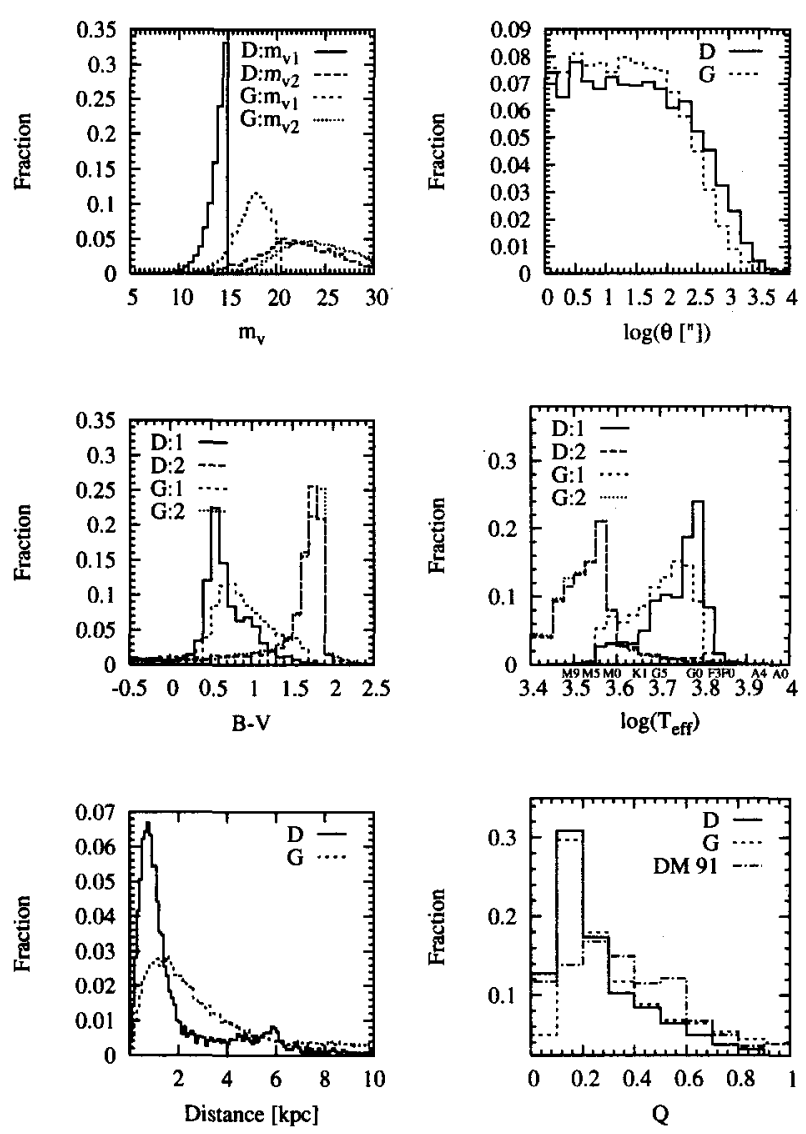

Fig. 1.

\section{RESULTS}

To select a binary population for DIVA, we restrict the GSC sample only to stars that have $m_{v}<15$, which is roughly the observational limit for DIVA. The limit is larger for late type stars, but since the most likely primary is a G-type star, we do not include any color dependence in the analysis. For GAIA double stars we have used the whole GSC catalog. The GSC 2.2 catalog is complete down to 18.5 in photographic $\mathrm{F}$ and 19.5 in photographic $\mathrm{J}$. In the figures D refers to DIVA magnitude limit and $\mathrm{G}$ refers GAIA magnitude limit i.e. the full GSC 2.2 catalog.

In Figure 1 (the upper left panel) $m_{v}$ distributions are shown for primary and secondary stars $\left(m_{v 1}\right.$ and $\left.m_{v 2}\right)$. Primary star magnitude distributions are almost the same as the overall average magnitude distributions down the $m_{v}$ limit in the
GSC. Secondary star distributions are very wide and a large fraction of low-mass binaries are beyond the observational capabilities of the missions.

For DIVA magnitude limits, the magnitude difference distribution is centered at 7 and for GAIA limits the maximum is at $\sim 6$ magnitudes. The detection of the binaries having large magnitude differences will be a great challenge to both missions. The upper right panel shows the mutual separation $\theta$ distributions. The upper limit in the distributions is due to the fact that the maximum semi-major axis is set to $10^{6} \mathrm{AU}$. Since the field of view is small in both missions and a large fraction of secondaries are fainter than detection thresholds, only a small fraction of all the binaries will be detected. We also obtain the color (B-V) and effective temperature $T_{\text {eff }}$ distributions for the binary components (middle left and right panels, respectively). Primary and secondary star distributions are well separated, since a typical primary star is a G-type dwarf and M-type star is the most common for the secondaries. Distance distributions and mass-ratio $Q$ distributions are given in the last two panels. Naturally, down to the GAIA magnitude limit there are much more distant stars than for DIVA limit. The most common distance down to the DIVA limit is $\sim 700 \mathrm{pc}$ and $\sim 1.5 \mathrm{kpc}$ for GAIA limit. The mass ratio distribution comes from the observed present day mass function, obtained from the simulation, combined with the present day mass function from which the secondary star is derived. In the lower right panel, also the observed $Q$-distribution for G-type dwarfs is given (DM91: Duquennoy \& Mayor 1991).

The Guide Star Catalogue-II is a joint project of the Space Telescope Science Institute and the Osservatorio Astronomico di Torino. This project was supported by the ESA-PRODEX contract "From HIPPARCOS to GAIA" with no. $14847 / 00 / \mathrm{NL} / \mathrm{SFe}(\mathrm{IC})$.

\section{REFERENCES}

Duquennoy, A., \& Mayor, M. 1991, A\&A, 248, 485

Hurley, J. R., Pols, O. R., \& Tout, C. A. 2000, MNRAS, 315,543

Kroupa, P. 2002, Science, 295, 82

Nurmi, P., \& Boffin, H. M. J. 2003, A\&A, 408, 803 